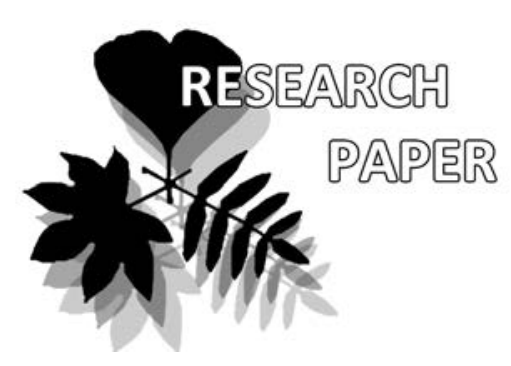

\title{
Diversity of fungal communities associated with mixotrophic pyroloids (Pyrola rotundifolia, $P$. media and Orthilia secunda) in their natural habitats
}

\author{
Ekaterina F. Malysheva ${ }^{1 *}$, Vera F. Malysheva ${ }^{1}$, \\ Elena Yu. Voronina ${ }^{2} \&$ Alexander E. Kovalenko $^{1}$
}

Ekaterina F. Malysheva ${ }^{1 *}$

e-mail: e_malysheva@binran.ru

Vera F. Malysheva

e-mail: v_malysheva@binran.ru

Elena Yu. Voronina ${ }^{2}$

e-mail:mvsadnik@list.ru

Alexander E. Kovalenko ${ }^{1}$

e-mail: alkov@binran.ru

${ }^{1}$ Komarov Botanical Institute of the Russian Academy of Sciences, Saint-Petersburg, Russia

${ }^{2}$ Lomonosov Moscow State University, Moscow, Russia

\section{* corresponding author}

Manuscript received: 14.05.2018 Review completed: 03.07.2018

Accepted for publication: 11.07.2018

Published online: 13.07.2018

\begin{abstract}
A B S T R A C T
As it was shown, mixotrophic plants (MxP) strongly depend on their mycorrhizal fungi for carbon, at least at the early stages of life cycle, and have rather high specificity for mycobionts. However, the diversity of fungi associated with MXP and their role in plant's life are still poorly known, especially under natural conditions. In the present study, the diversity of mycobionts of the three mixotrophic pyroloid species (Pyrola rotundifolia, P. media and Orthilia secunda) was investigated by sequencing $n r$ TS from roots and rhizomes. At the same time, we studied ectomycorrhizal fungal communities of neighboring trees. The mycobiont diversity slightly differed berween the three species, but they also shared similar fungal taxa. The species of basidiomycete genera Tomentella, Piloderma, Russula and Mycena were dominant fungal partners of the studied pyroloids. The plants were also colonized by other ectomycorrhizal and saprotrophic basidiomycetes and ascomycetes. The research results showed MxP link to tree species by shared mycobionts and partial mycoheterotrophy by involvement into mycelial network. Thirty nine fungal taxa (at species and genera level) inhabiting pyroloid root system as mycobionts and root endophytes were detected. Their role for plant performance requires further investigation.
\end{abstract}

K e y w o r d s : Pyroleae, arbutoid mycorrhiza, mixotrophy, $n r$ ITS, operational taxonomic units, symbionts, endophytes

\section{P E 3 Ю M E}

Малышева Е.Ф., Малышева В.Ф., Воронина Е.Ю., Коваленко А.Е. Разнообразие сообществ грибов, связанных с миксотрофными грушанковыми (Pyrola rotundifolia, P. media и Orthilia secunda) в их естественных местообитаниях. Миксотрофные растения (МхР) сильно зависят в углеродном питании от микоризных грибов, по крайней мере, на ранних стадиях развития, и облаАают высокой специфичностью к микобионтам. ОАнако еще Аовольно мало известно о разнообразии грибов, связанных с MхР, и их роли в жизни растений, особенно в естественных экосистемах. В настоящей работе было исследовано разнообразие микобионтов трех виАов грушанковых с миксотрофным типом питания (Pyrola rotundifolia, P. media и Orthilia secunda) путем вылемения $А Н К$ и последующего секвенирования $n r \mathrm{ITS}$ из корней и корневищ. ОАновременно изучался состав грибов, формирующих эктомикоризу с соседними Аеревьями. Обнаруженные сообщества микобионтов немного отличались у трех виАов грушанковых, хотя были выявлены общие симбионты. Аоминирующими партнерами в арбутоидной микоризе изученных виАов растений явиАись базидиомицеты из родов Tomentella, Piloderma, Russula и Mycena. Растения были также колонизированы Аругими эктомикоризными и сапротрофными базидиомицетами и аскомицетами. Результаты исследования показали связь МхР с Аревесными растениями в природе благодаря наличию общих микобионтов, с помощью которых растения вовлекаются в единую мицелиальную сеть. Всего было выяв ено 39 грибных таксонов (видового и родового рангов), обитающих в корневой системе грушанковых в качестве микобионтов и корневых эндофитов. Их роль в жизни растений требует Аальнейшего изучения.

КАючевые слова: Pуroleae, арбутоидная микориза, миксотрофия, $n$ ITS, операционные таксономические единицы, симбионты, эндофиты
Phylogeny and physiology of pyroloid plants (members of tribe Pyroleae) have attracted great interest from scientists for more than 200 years (Hynson et al. 2009, 2013). One of the problems with the group's taxonomy is the presence of both leafless (potentially mycoheterotrophic plants) and transitional to fully autotrophic forms in the genus Pyrola L. As a result of recent studies based on molecular data (Freudenstein 1999, Kron et al. 2002, Liu et al. 
2010), pyroloids have come to be treated as a separate tribe (Pyroleae) in the subfamily Monotropoideae of Ericaceae. One of the essential features of these plants is the production of a vast number of dust-like seeds (the phenomenon of microspermy) with a minimum nutrient supply. It is known that this trait (dust-like seeds) is also typical for many plants, from at least 12 different families including orchids, and has emerged independently in different phylogenetic lines (Eriksson \& Kainulainen 2011). Because of limited carbon reserve such seeds evidently strongly depend on the external carbon sources for their germination, and they compensate for this deficiency by symbiotic connections with fungi.

This nutritional mode was termed "initial mycoheterotrophy” (Leake 1994, Merckx 2013). It is noteworthy that all species of Pyrola are initially mycoheterotrophic at the early stages of ontogeny but upon reaching full maturity they demonstrate practically the complete range of trophic strategies, from obligate autotrophy to full mycoheterotrophy (e.g. achlorophyllous Pyrola aphylla Smith) including transitional forms. The phenomenon of mixotrophy (or partial mycoheterotrophy) is inherent in such transitional forms. It consists in the possibility of simultaneous use of two independent sources of carbon by adult green plants - one as a product of photosynthesis, and another coming through a mycorrhizal association with fungi (Tedersoo et al. 2007, Zimmer et al. 2007, Selosse \& Roy 2009, Matsuda et al. 2012). The mixotrophic plants were shown to demonstrate $\delta^{13} \mathrm{C}$ and $\delta^{15} \mathrm{~N}$ values which are the average between these indices typical for fully mycoheterotrophic and autotrophic plants (Hynson et al. 2009). However, the $\delta^{13} \mathrm{C}$ value in mixotrophic pyroloids, as in orchids, may depend on the stage of development, type of habitat, lighting conditions and other factors (Bidartondo et al. 2004, Tedersoo et al. 2007, Zimmer et al. 2007, Johansson et al. 2015). Moreover, it was observed that the same Pyrola species occurring in different geographic regions significantly vary in the degree of autoand heterotrophy, and the causes of this variability have not yet been definitively elucidated (Tedersoo et al. 2007, Zimmer et al. 2007, Hynson et al. 2009).

The tribe Pyroleae contains four genera (Pyrola, Ortbilia Raf., Chimaphila Pursh and Moneses Salisb. ex Gray) and about 40 species of herbs and subshrubs distributed in the Northern Hemisphere that usually grow in forests under the canopy of trees. The pyroloids form an arbutoid mycorrhiza, in which both intracellular and external (mantle) fungal structures are normally formed, and the morphology of the mycorrhizal root tips can be modified, similar to that occurring in ectomycorhizal tree root tips (Robertson \& Robertson 1985, Smith \& Read 2008). Arbutoid mycorrhiza is poorly known compared to other types of mycorrhizal symbiosis but it is crucial for forest plant communities by providing joint of tree and tree-shrub layers.

The ectomycorrhizal mycobionts can act as fungal partners in for pyroloids thus allowing the formation of mycelial networks linking plants of tree, shrub and herb layers in forest communities (Tedersoo et al. 2007, Zimmer et al. 2007, Massicotte et al. 2008, Vincenot et al. 2008, Hynson \& Bruns 2009, Toftegaard et al. 2010, Hashimoto et al. 2012).
Such associations have important environmental consequences: pyroloids growing under the forest canopy are provided with additional source of carbon in the form of ectomycorrhizal woody plants ' photosynthetic products, but for their part serve as a "depot" of the mycobiont, which is essential when the tree layer is restored after disturbances caused, for example, by clear-cutting (Smith \& Read 2008).

Until the present, not much efforts has been devoted to studying the phenomenon of mixotrophy in pyroloids as compared to the orchids (Matsuda et al. 2012). The subject of study was and remains: a study of seed germination physiology; revealing the diversity of fungi forming mycorrhiza with plants at different stages of ontogenesis as well as the specificity of these symbionts for different species from Pyroleae; a study of changes in plant trophic strategies under different environmental conditions. Initially it was supposed, and then there was evidence (Hynson \& Bruns 2009, Hashimoto et al. 2012) that all pyroloids (like orchids) are very demanding for the specific composition of the ectomycorrhizal fungi, but, unlike other representatives of Monotropoideae, not throughout the entire life cycle, but only in the early stages of their development. However, the accumulated data is still insufficient to talk about the direct dependence of mixotrophic plant on fungi, even for the most widespread species of pyroloids. There are still a lot of studies to be done in this direction, and the work presented herein is one of them.

The present research addresses the study of mycorrhizal colonization of three pyroloid species (Pyrola rotundifolia L., P. media Sw. and Orthilia secunda (L.) House) widely distributed in coniferous and mixed forests of the European part of Russia. Data on their mycorrhizas are limited and vary considerably depending on the area of study and the type of plant communities.

Several populations of plants growing in different habitats were studied. Identification of fungal mycobionts using molecular techniques was carried out.

\section{MATERIAL AND METHODS}

\section{Study site}

A total of 67 pyroloid plants at different developmental stages from 16 populations and more than 100 mycorrhizal root tips of surrounding trees were sampled from 5 sites $(50 \times 50 \mathrm{~m})$ in European part of Russia in 2016-2017 (from July to September). The studied populations of pyroloid species were at least $10 \mathrm{~m}$ apart. All sites are located in temperate climatic zone and represent natural forests or forest plantation. They differ in environmental conditions and plant community composition. Detailed information on the study sites is shown in Table 1.

\section{Sampling procedure and preparation for DNA extraction}

At each site, 3-4 plants from each population were randomly selected. Additionally, ectomycorrhizal root tips of surrounding trees were collected in accordance with previously described procedure (Malysheva et al. 2016). The fruitbodies of ectomycorrhizal macromycete species were sampled at the study sites to provide references for spe- 
Table 1. Characteristics of study sites.

\begin{tabular}{|l|l|l|l|l|l|}
\hline $\begin{array}{l}\text { Study } \\
\text { site }\end{array}$ & Location & $\begin{array}{l}\text { Latitude/ } \\
\text { longitude }\end{array}$ & Plant community & $\begin{array}{l}\text { Ectomycorrhizal trees in the } \\
\text { habitat }\end{array}$ & Pyroloid species \\
\hline S1 & $\begin{array}{l}\text { Moscow Region, } \\
\text { Zvenigorod Biological } \\
\text { Station, square 6 }\end{array}$ & $\begin{array}{l}55^{\circ} 41^{\prime} 28 \mathrm{~N}, \mathrm{~N}, \\
36^{\circ} 43^{\prime} 06 \mathrm{E}\end{array}$ & Coniferous forest & $\begin{array}{l}\text { Picea abies, Pinus sylvestris, Betula sp., } \\
\text { Quercus robur, Sorbus aucuparia, Tilia } \\
\text { cordata }\end{array}$ & $\begin{array}{l}\text { Pyrola rotundifolia, } \\
\text { Pyrola media, } \\
\text { Ortbilia secunda }\end{array}$ \\
\hline S2 & $\begin{array}{l}\text { Moscow Region, } \\
\text { Zvenigorod Biological } \\
\text { Station, square 7 }\end{array}$ & $\begin{array}{l}55^{\circ} 41^{\prime} 28^{\prime \prime} \mathrm{N}, \\
36^{\circ} 43^{\prime} 46^{\prime \prime} \mathrm{E}\end{array}$ & Mixed forest & $\begin{array}{l}\text { Betula sp., Pinus sylvestris, Quercus ro- } \\
\text { bur, Sorbus aucuparia, Tilia cordata, Po- } \\
\text { pulus tremula, Salix caprea, Picea abies }\end{array}$ & $\begin{array}{l}\text { Pyrola rotundifolia, } \\
\text { Pyrola media }\end{array}$ \\
\hline S3 & $\begin{array}{l}\text { Moscow Region, } \\
\text { Zvenigorod Biological } \\
\text { Station, square 20 }\end{array}$ & $\begin{array}{l}55^{\circ} 41^{\prime} 06^{\prime \prime} \mathrm{N}, \\
36^{\circ} 43^{\prime} 54 " \mathrm{E}\end{array}$ & Mixed forest & $\begin{array}{l}\text { Picea abies, Pinus sylvestris, Betula } \\
\text { sp., Quercus robur, Sorbus aucuparia, } \\
\text { Populus tremula, Acer platanoides }\end{array}$ & $\begin{array}{l}\text { Pyrola media, } \\
\text { Ortbilia secunda }\end{array}$ \\
\hline S4 & $\begin{array}{l}\text { Samara Region, vicinities } \\
\text { of Pribrezhny }\end{array}$ & $\begin{array}{l}53^{\circ} 29^{\prime} 46^{\prime \prime} \mathrm{N} \\
49^{\circ} 52^{\prime} 51^{\prime \prime} \mathrm{E}\end{array}$ & $\begin{array}{l}\text { Pine forest } \\
\text { plantation (50-60 } \\
\text { years of age) }\end{array}$ & $\begin{array}{l}\text { Pinus sylvestris, Populus tremula, Tilia } \\
\text { cordata }\end{array}$ & Ortbilia secunda \\
\hline $\begin{array}{l}\text { Samara Region, Zhiguli } \\
\text { Nature Reserve, vicinities } \\
\text { of Gudronny }\end{array}$ & $\begin{array}{l}53^{\circ} 23^{\prime} 15^{\prime \prime} \mathrm{N} \\
49^{\circ} 45^{\prime} 44^{\prime \prime} \mathrm{E}\end{array}$ & Birch forest & $\begin{array}{l}\text { Betula pendula, Tilia cordata, Sorbus } \\
\text { aucuparia, Salix caprea, Acer } \\
\text { platanoides, Pinus sylvestris }\end{array}$ & Ortbilia secunda \\
\hline
\end{tabular}

cies revealed from the root tips examined. The plant root samples with adhering soil were wrapped with polyethylene film or aluminum foil and placed into sealed plastic bags for transporting to the laboratory. During transport and prior to processing, samples were kept moist and stored in the dark at $4^{\circ} \mathrm{C}$. In the laboratory, they were thoroughly washed with tap water and processed within 3 days of sampling. Then roots of pyroloids and trees were treated in the same way.

The collected root samples were divided into segments (5-20 mm length). Individual segments of roots and rhizomes that appeared to be colonized by fungi were placed in $2 \%$ cetyltrimethylammonium bromide (CTAB) buffer in $1.5 \mathrm{ml}$ tubes for further analysis.

\section{DNA extraction, PCR amplification and sequencing}

Fungal DNA was extracted from roots and rhizomes of pyroloids and ectomycorrhizal tips using the Nucleo-Spin Plant II Kit (Macherey-Nagel GmbH \& Co. KG), according to the manufacturer's instructions. The fungal $n$ rTS region (ITS1-5.8S-ITS2) was amplified using two sets of primers: ITS1F + ITS4 and ITS1F + ITS4B (Gardes \& Bruns 1993, White et al. 1990). Successful amplifications confirmed visually with agarose gel electrophoresis were then cleaned using the GeneJET PCR Purification Kit (Thermo Scientific, Thermo Fisher Scientific Inc., MA, USA) and quantified using the NanoPhotometer P-300 (Implen GmbH, Germany). In the case of two or more bands amplified, the total product was loaded on agarose gel and then each band was extracted from gel and recovered using the GeneJET Gel Extraction Kit (Thermo Scientific, Thermo Fisher Scientific Inc., MA, USA), according to the manufacturer's protocol for gel extraction. The prepared PCR product was then sequenced with the same primer pairs. DNA sequencing was performed on an ABI3130 Genetic Analyzer using BigDye ver. 3.1 chemistry (Applied Biosystems, Foster City, CA, USA). Electrophoretograms were checked using Sequencing Analysis 5.3.1 (Applied Biosystems) and MEGA 6 (Tamura et al. 2013).

All stages of molecular studies were carried out on equipment of the Center for collective use of scientific equipment "Cellular and molecular technology of studying plants and fungi" at the Komarov Botanical Institute of the Russian Academy of Sciences.

\section{Identification of fungal taxa and phylogenetic analysis}

Obtained sequences with high quality and degree of similarity (cutoff of $97 \%$ ) were clustered into groups and were manually processed and optimized using the MEGA 6. Chimeric sequences assessed by reference-based checking using GenBank were removed from further analyses. We regarded a sequence as a chimera when its ITS1 and ITS2 regions had $98-100 \%$ similarity to different species or $>90 \%$ similarity to different genera. The remaining consensus sequences were then analyzed to ascertain taxonomic affinity. Identification of "Operational Taxonomic Units" (OTUs) was based on the BLASTn search algorithm in NCBI GenBank (http:/ /www.ncbi.nlm.nih.gov/genbank/) or UNITE sequence databases (http:/ / unite.ut.ee/, Kõljalg et al. 2005). The degree of similarity was accepted: for species level $\geq 97 \%$ (Kõljalg et al. 2013, Smith et al. 2013), for genus level $95-97 \%$ and for family to order level $<95 \%$. When two or more sequences or sequence groups differed to each other but matched the same entry in databases, a number was added to the name to mark the differences (e.g. Tomentella sp. 1 and Tomentella sp. 2). A list with the taxa names and the accession numbers of the corresponding database entry of the best match is given in Table 2 . All newly generated sequences have been deposited in GenBank with corresponding accession numbers.

The fungal sequences generated from pyroloids were used to construct phylogenetic tree. Phylogenetic analysis was carried out separately for Ascomycota and Basidiomycota. The closest-matching sequences from the GenBank and UNITE databases were additionally involved into phylogenetic analyses. For both datasets the sequences were aligned with the Muscle (Edgar 2004) embedded into MEGA 6. The alignments were adjusted manually using the same program. Phylogenetic reconstructions for data sets were performed with maximum likelihood (ML) analyses in the PhyML server, v. 3.0 (http://www.atgc-montpellier.fr/phyml/), with one hundred rapid bootstrap replicates under GTR model. 
Table 2. Fungal diversity associated with three pyroloid species (Pyrola rotundifolia, Pyrola media and Ortbilia secunda) and surrounding trees at five study sites.

\begin{tabular}{|c|c|c|c|c|c|c|}
\hline Taxa & $\begin{array}{l}\text { Putative } \\
\text { ecology }\end{array}$ & Host plant & $\begin{array}{l}\text { Study } \\
\text { site }\end{array}$ & $\begin{array}{l}\text { GenBank } \\
\text { accession } \\
\text { no. }\end{array}$ & $\begin{array}{l}\text { Best BLASTn match (accession no. of } \\
\text { GenBank or UNITE) }\end{array}$ & $\begin{array}{l}\text { Simi- } \\
\text { larity } \\
(\%)\end{array}$ \\
\hline \multicolumn{7}{|l|}{ Basidiomycota } \\
\hline $\begin{array}{l}\text { Amanita pantherina } \\
\text { Amphinema byssoides }\end{array}$ & $\begin{array}{l}\text { EcM } \\
\text { EcM }\end{array}$ & $\begin{array}{l}\text { Pinus sylvestris } \\
\text { Picea abies }\end{array}$ & $\begin{array}{l}4 \\
1\end{array}$ & $\begin{array}{l}\text { MF926541 } \\
\text { MF92654, } \\
\text { MF926543 }\end{array}$ & $\begin{array}{l}\text { Amanita pantherina AB080774, AB080776 } \\
\text { Amphinema byssoides JN943928 }\end{array}$ & $\begin{array}{l}97 \\
99\end{array}$ \\
\hline $\begin{array}{l}\text { Bjerkandera adusta } \\
\text { Cortinarius cf. disjungendus } \\
\text { Cortinarius hemitrichus } \\
\text { Echinoderma asperum } \\
\text { Gerbardtia borealis } \\
\text { Hebeloma sp. }\end{array}$ & $\begin{array}{l}\text { Sap } \\
\text { EcM } \\
\text { EcM } \\
\text { Sap } \\
\text { Sap } \\
\text { EcM }\end{array}$ & $\begin{array}{l}\text { P. rotundifolia } \\
\text { Tilia cordata } \\
\text { P. media } \\
\text { P. media } \\
\text { O. secunda } \\
\text { Populus tremula }\end{array}$ & $\begin{array}{c}2,3 \\
5 \\
2 \\
3 \\
1 \\
5\end{array}$ & $\begin{array}{l}\text { MH270625 } \\
\text { MF926544 } \\
\text { MH270626 } \\
\text { MF926545 } \\
\text { MH270627 } \\
\text { MF926546 }\end{array}$ & $\begin{array}{l}\text { Bjerkandera adusta KC176315 } \\
\text { Cortinarius disjungendus KP013191 } \\
\text { Cortinarius bemitrichus DQ } 097870 \\
\text { Lepiota aspera AY176354 } \\
\text { Gerbardtia borealis KP858004 } \\
\text { Hebeloma subconcolor KT218391 } \\
\text { Hebeloma leucosarx KT218361 }\end{array}$ & $\begin{array}{c}99 \\
95 \\
100 \\
98 \\
99 \\
99\end{array}$ \\
\hline Laccaria proxima & $\mathrm{EcM}$ & Picea abies & 1 & MF926547 & $\begin{array}{l}\text { Laccaria proxima KM067833 } \\
\text { Laccaria proxima IX907813 }\end{array}$ & 99 \\
\hline $\begin{array}{l}\text { Lactarius cf. tabidus } \\
\text { Lactarius torminosus } \\
\text { Lentinus crinitus } \\
\text { Luellia recondita } \\
\text { Mycena } \mathrm{cf} \text {. cinerella }\end{array}$ & $\begin{array}{c}\text { EcM } \\
\text { EcM } \\
\text { Sap } \\
\text { PEcM/Sap } \\
\text { Sap }\end{array}$ & $\begin{array}{l}\text { Picea abies } \\
\text { P. media } \\
\text { P. media, O. secunda } \\
\text { O. secunda } \\
\text { O. secunda, Pinus } \\
\text { sylvestris }\end{array}$ & $\begin{array}{l}3 \\
2 \\
3 \\
3 \\
1\end{array}$ & $\begin{array}{l}\text { MH270628 } \\
\text { MH270629 } \\
\text { MF926548 } \\
\text { MF926550 } \\
\text { MF926553, } \\
\text { MF926554 }\end{array}$ & $\begin{array}{l}\text { Lactarius cf. tabidus LK932113 } \\
\text { Lactarius torminosus DQ367908 } \\
\text { Lentinus crinitus GU207289 } \\
\text { Luellia recondita JF519370 } \\
\text { Mycena cinerella KT900146 }\end{array}$ & $\begin{array}{l}96 \\
98 \\
97 \\
98 \\
98\end{array}$ \\
\hline $\begin{array}{l}\text { Mycena cf. citrinomarginata } \\
\text { Mycena galopus } \\
\text { Phanerochaete chrysosporum } \\
\text { Piloderma bicolor } \\
\text { Piloderma sphaerosporum }\end{array}$ & $\begin{array}{l}\text { Sap } \\
\text { Sap } \\
\text { Sap } \\
\text { EcM } \\
\text { EcM }\end{array}$ & $\begin{array}{l}\text { P. media } \\
\text { O. secunda } \\
\text { P. media } \\
\text { P. media } \\
\text { O. secunda, Picea } \\
\text { abies }\end{array}$ & $\begin{array}{l}2 \\
3 \\
3 \\
1 \\
1\end{array}$ & $\begin{array}{l}\text { MF926551 } \\
\text { MF926552 } \\
\text { MF926555 } \\
\text { MF926556 } \\
\text { MF926557, } \\
\text { MF926558, } \\
\text { MF926559 }\end{array}$ & $\begin{array}{l}\text { Mycena citrinomarginata KJ705180 } \\
\text { Mycena galopus KU516420 } \\
\text { Phanerochaete chrysosporium KM } 277996 \\
\text { Piloderma bicolor KP814514 } \\
\text { Piloderma sphaerosporum JQ711814 }\end{array}$ & $\begin{array}{l}95 \\
97 \\
97 \\
98 \\
100\end{array}$ \\
\hline $\begin{array}{l}\text { Pleurotus ostreatus } \\
\text { Pseudotomentella tristis } \\
\text { Russula aeruginea } \\
\text { Russula consobrina } \\
\text { Russula font-queri } \\
\text { Russula intermedia } \\
\text { Russula laricina } \\
\text { Russula cf. subfoetens } \\
\text { Russula velenovskyi }\end{array}$ & $\begin{array}{l}\text { Sap } \\
\text { EcM } \\
\text { EcM } \\
\text { EcM } \\
\text { EcM } \\
\text { EcM } \\
\text { EcM } \\
\text { EcM } \\
\text { EcM }\end{array}$ & $\begin{array}{l}\text { O. secunda } \\
\text { O. secunda } \\
\text { Pinus sylvestris } \\
\text { P. rotundifolia } \\
\text { Pinus sylvestris } \\
\text { Picea abies } \\
\text { Pinus sylvestris } \\
\text { P. rotundifolia } \\
\text { O. secunda, Tilia } \\
\text { cordata }\end{array}$ & $\begin{array}{l}3,5 \\
3 \\
5 \\
1 \\
3 \\
4 \\
2 \\
5\end{array}$ & $\begin{array}{l}\text { MF926560 } \\
\text { MH270630 } \\
\text { MF926562 } \\
\text { MF926563 } \\
\text { MF926561 } \\
\text { MH270631 } \\
\text { MF926568 } \\
\text { MH270632 } \\
\text { MF926564 }\end{array}$ & $\begin{array}{l}\text { Pleurotus ostreatus LC149608 } \\
\text { Pseudotomentella tristis KY681465 } \\
\text { Russula aeruginea KX095030 } \\
\text { Russula consobrina JF908696 } \\
\text { Russula font-queri KT934003, KU949378 } \\
\text { Russula intermedia UDB019791 } \\
\text { Russula laricina KF850405 } \\
\text { Russula cf. subfoetens KF245511 } \\
\text { Russula velenovskyi JQ888202 }\end{array}$ & $\begin{array}{l}99 \\
99 \\
97 \\
97 \\
99 \\
98 \\
100 \\
98 \\
99\end{array}$ \\
\hline Russula cf. versicolor & EcM & Betula pendula & 5 & $\begin{array}{l}\text { MF926565, } \\
\text { MF926566 }\end{array}$ & Russula versicolor JQ711937 & 99 \\
\hline $\begin{array}{l}\text { Russula sp. } \\
\text { Tomentella stuposa }\end{array}$ & $\begin{array}{l}\text { EcM } \\
\text { EcM }\end{array}$ & $\begin{array}{l}\text { Pinus sylvestris } \\
\text { Picea abies, } \\
\text { O. secunda }\end{array}$ & $\begin{array}{l}4 \\
1\end{array}$ & $\begin{array}{l}\text { MF926567 } \\
\text { MF926571, } \\
\text { MH270637 }\end{array}$ & $\begin{array}{l}\text { Russula laricina KF850405 } \\
\text { Tomentella stuposa JQ888213 }\end{array}$ & $\begin{array}{l}95 \\
99\end{array}$ \\
\hline $\begin{array}{l}\text { Tomentella sp.1 } \\
\text { Tomentella sp.2 }\end{array}$ & $\begin{array}{l}\text { EcM } \\
\text { EcM }\end{array}$ & $\begin{array}{l}\text { O. secunda } \\
P . \text { rotundifolia, } \\
O \text {. secunda }\end{array}$ & 2,3 & $\begin{array}{l}\text { MF926573 } \\
\text { MF926574, } \\
\text { MF926575 }\end{array}$ & $\begin{array}{l}\text { Tomentella sp. AJ534915 } \\
\text { Tomentella sublilacina HM189985 }\end{array}$ & $\begin{array}{l}99 \\
95\end{array}$ \\
\hline $\begin{array}{l}\text { Tomentella sp. } 3 \\
\text { Thelephora cf. anthocephala }\end{array}$ & $\begin{array}{l}\text { EcM } \\
\text { EcM }\end{array}$ & $\begin{array}{l}\text { P. rotundifolia } \\
\text { O. secunda, Populus } \\
\text { tremula }\end{array}$ & $\begin{array}{l}1 \\
5\end{array}$ & $\begin{array}{l}\text { MH270633 } \\
\text { MF926569, } \\
\text { MF926570 }\end{array}$ & $\begin{array}{l}\text { Tomentella sp. JQ711817 } \\
\text { Thelephora anthocephala KP454019 }\end{array}$ & $\begin{array}{l}98 \\
95\end{array}$ \\
\hline Tylospora fibrillosa & $\mathrm{EcM}$ & O. secunda & 3 & MF926576 & Tylospora fibrillosa JQ711996 & 98 \\
\hline $\begin{array}{l}\text { Ascomycota } \\
\text { Acremonium sp. } \\
\text { Aureobasidium sp. } \\
\text { Cadophora finlandica }\end{array}$ & $\begin{array}{c}\text { Sap } \\
\text { Sap } \\
\text { Endo, } \\
\text { PEcM, Eric }\end{array}$ & $\begin{array}{l}\text { P. rotundifolia } \\
\text { O. secunda } \\
\text { P. media }\end{array}$ & $\begin{array}{l}1 \\
4 \\
3\end{array}$ & $\begin{array}{l}\text { MF926521 } \\
\text { MF926522 } \\
\text { MF926523 }\end{array}$ & $\begin{array}{l}\text { Acremonium sp. KU } 376503 \text {, LT598644 } \\
\text { Aureobasidium pullulans KX067792, KX } 444670 \\
\text { Cadophora finlandica EF093179 }\end{array}$ & $\begin{array}{l}97 \\
90 \\
97\end{array}$ \\
\hline $\begin{array}{l}\text { Chrysomyxa pyrolae } \\
\text { Cladosporium cladosporoides } \\
\text { Helvella sp. } \\
\text { Humaria bemisphaerica }\end{array}$ & $\begin{array}{l}\text { Pathog } \\
\text { Sap } \\
\text { EcM } \\
\text { EcM }\end{array}$ & $\begin{array}{l}\text { P. media } \\
\text { P. rotundifolia } \\
\text { O. secunda } \\
\text { O. secunda }\end{array}$ & $\begin{array}{c}3 \\
2 \\
5 \\
1,4\end{array}$ & $\begin{array}{l}\text { MF926524 } \\
\text { MH270634 } \\
\text { MF926525 } \\
\text { MF926526, } \\
\text { MF926527, } \\
\text { MF926528 }\end{array}$ & $\begin{array}{l}\text { Chrysomyxa pyrolae GU049485 } \\
\text { Cladosporium cladosporoides MF077224 } \\
\text { Helvella sp. KM576410 } \\
\text { Humaria hemisphaerica DQ200832 }\end{array}$ & $\begin{array}{l}99 \\
98 \\
99 \\
98\end{array}$ \\
\hline $\begin{array}{l}\text { Ilyonectria sp. } \\
\text { Oidiodendron maius }\end{array}$ & $\begin{array}{c}\text { Pathog } \\
\text { Eric }\end{array}$ & $\begin{array}{l}\text { P. rotundifolia } \\
\text { O. secunda }\end{array}$ & $\begin{array}{l}3 \\
4\end{array}$ & $\begin{array}{l}\text { MH270635 } \\
\text { MF926533, } \\
\text { MF926534 }\end{array}$ & $\begin{array}{l}\text { Ilyonectria sp. KU556513 } \\
\text { Oidiodendron maius AF062800, AB089654 }\end{array}$ & $\begin{array}{l}100 \\
97\end{array}$ \\
\hline $\begin{array}{l}\text { Pezoloma ericae } \\
\text { Phialocephala europaea } \\
\text { Phialocephala fortinii }\end{array}$ & $\begin{array}{l}\text { Eric } \\
\text { Endo } \\
\text { Endo }\end{array}$ & $\begin{array}{l}\text { Pinus sylvestris } \\
\text { O. secunda } \\
\text { O. secunda, Picea } \\
\text { abies }\end{array}$ & $\begin{array}{c}1 \\
4 \\
4,1\end{array}$ & $\begin{array}{l}\text { MF926532 } \\
\text { MF926529 } \\
\text { MF926530, } \\
\text { MF926531 }\end{array}$ & $\begin{array}{l}\text { Rhizoscyphus ericae JQ711893 } \\
\text { Phialocephala europaea AY078137 } \\
\text { Pbialocephala fortinii KX440138 }\end{array}$ & $\begin{array}{l}97 \\
99 \\
98\end{array}$ \\
\hline $\begin{array}{l}\text { Sphaerosporella sp. } \\
\text { Thuemenidium } \\
\text { atropurpureum }\end{array}$ & $\begin{array}{l}\text { PEcM } \\
\text { Sap/ } \\
\text { Unknown }\end{array}$ & $\begin{array}{l}\text { O. secunda } \\
\text { O. secunda, P. media }\end{array}$ & $4, \stackrel{1}{1}, 3$ & $\begin{array}{l}\text { MH270636 } \\
\text { MF926535, } \\
\text { MF926536, } \\
\text { MF926537, } \\
\text { MF926538, } \\
\text { MF926539 }\end{array}$ & $\begin{array}{l}\text { Sphaerosporella sp. JQ711781 } \\
\text { Thuemenidium atropurpureum JQ256427 }\end{array}$ & $\begin{array}{l}97 \\
99\end{array}$ \\
\hline $\begin{array}{l}\text { Tuber pacificum } \\
\text { Wilcoxina rebmii }\end{array}$ & $\begin{array}{c}\text { EcM } \\
\text { EEnM }\end{array}$ & $\begin{array}{l}\text { O. secunda } \\
\text { O. secunda }\end{array}$ & $\begin{array}{r}5 \\
3,4,5\end{array}$ & $\begin{array}{l}\text { MF926540 } \\
\text { MF926517, } \\
\text { MF926518, } \\
\text { MF926519, } \\
\text { MF926520 }\end{array}$ & $\begin{array}{l}\text { Tuberpacificum JQ712002 } \\
\text { UNITE Species Hypotheses Wilcoxina rehmii } \\
\text { JQ975970 }\end{array}$ & $\begin{array}{l}98 \\
99\end{array}$ \\
\hline
\end{tabular}

EcM - ectomycorrhizal, EEnM - ectendomycorrhizal, PEcM - putative ectomycorrhizal, Eric - ericoid mycorrhizal, Sap - saprotrophic, Endo - endophytic, Pathog - pathogenic, Unknown - with unknown trophic status 


\section{Determination of OTUs trophic status}

The putative trophic status of the detected fungal OTUs was assigned based on a critical review of available literature (Massicotte et al. 1993, Erland 1995, Rice \& Currah 2006, Gorfer et al. 2007, Tedersoo et al. 2007, 2010).

\section{RES U LT S}

In total, 220 fungal DNA samples were obtained from the roots and rhizomes of studied pyroloid plants and 110 from the ectomycorrhizal root tips of surrounding trees. From pyroloids only 120 fungal $n r$ ITS sequences were generated and from trees -45 sequences. In our work we obtained a rather large number of heterogeneous PCR products (not included in subsequent analysis), that may be due to prevailing colonization of pyroloid's roots with numerous endophytes together with mycorrhizal symbionts, what was also noted for Pyroleae by other authors (Tedersoo et al. 2007, Vincenot et al. 2008). Overall, 52 OTUs of species or generic level were detected based on best BLASTn matches. The taxonomic hypotheses and putative ecology of revealed OTUs are provided in Table 2.

The ecology of recovered taxa associated with pyroloids did not significantly differ between the three species. Most of them represented ectomycorrhizal fungi (EcM, 17 OTUs), other were putative ectomycorrhizal (PEcM) or saprotrophic fungi (Sap, 17 OTUs), ectendomycorrhizal fungi (EEnM, 1 OTU), ericoid mycorrhizal fungi (Eric, 2 OTUs), and endophytes (Endo, 2 OTUs). The remaining taxa represented pathogenic fungi numbered 2 OTUs.

It is noticeable that most of the identified fungi belong to taxa from Basidiomycota (27 OTUs or $51.9 \%$ of total diversity) and Ascomycota (5 OTUs or $9.6 \%$ ) known as active participants of ectomycorrhizas with trees. At the family level following mycorrhizal and PEcM/saprotrophic fungi from the roots and rhizomes of the three species were identified: Thelephoraceae, Atheliaceae, Mycenaceae, Russulaceae, Phanerochaetaceae, Polyporaceae, Hydnodontaceae, Meruliaceae, Agaricaceae, Cortinariaceae, Pleurotaceae, Lyophyllaceae of Basidiomycota, and Pyronemataceae, Myxotrichaceae, Geoglossaceae, Saccotheciaceae, Tuberaceae, Helvellaceae, Vibrisseaceae, Cladosporiaceae of Ascomycota (and two representatives of Hypocreales and Helotiales with uncertain family affiliation) (Fig. 1).

Owing to their potentially pathogenic, endophytic or contaminant nature, the following taxa were not treated as mycorrhizal or putative mycorrhizal fungi and excluded from the phylogenetic analysis (Fig. 2): Chrysomyxa pyrolae, Ilyonectria sp., Acremonium sp. and Cladosporium sp.

The richest in the number of species were corticioid genera Tomentella and Piloderma, as well as agaricoid genera Russula and Mycena, which were commonly found in roots of both pyroloid plants and neighboring coniferous trees (mostly in Picea and Pinus roots). The fungal partner Tomentella sp. 2 was shared by Orthilia secunda and Pyrola rotundifolia, whereas Thuemenidium atropurpureum, Wilcoxina rehmii and Lentinus crinitus were common to Ortbilia secunda and Pyrola media (Fig. 2, 3). The mycobionts Piloderma sphaerosporum, Tylospora fibrillosa, Russula velenovskyi, Tomentella sp. 1, Pseudotomentella tristis, Thelephora $\mathrm{cf}$. anthocephala, Luellia recondita,
Lentinus crinitus, Mycena cf. cinerella, Mycena galopus, Pleurotus ostreatus, Gerhardtia borealis, Humaria hemisphaerica, Phialocephala europaea, P. fortinii, Oidiodendron maius, Sphaerosporella sp., Aureobasidium sp., Tuber pacificum and Helvella sp. were associated only with Orthilia secunda. The following fungi: Piloderma bicolor, Phanerochaete chrysosporium, Cortinarius hemitrichus, Lactarius torminosus, Echinoderma asperum, Mycena cf. citrinimarginata, Cadophora finlandica and Chrysomyxa pyrolae were associated with Pyrola media, but Russula consobrina, R. subfoetens, Tomentella sp.3, Bjerkandera adusta, Ilyonectria sp., Acremonium sp. and Cladosporium sp. were detected from Pyrola rotundifolia root system.

\section{I S C U S S I O N}

Molecular identification of fungi in the selected root fragments from Pyrola rotundifolia, P. media and Ortbilia secunda revealed a broad range of fungal taxa (39 OTUs). Among the mycobionts associated with the three species the group of Basidiomycota dominated and numbered 24 OTUs (62\%), followed by Ascomycota with 15 OTUs (38\%). The vast majority of OTUs detected belonged to EcM fungi. They are mostly represented by members of genera Tomentella, Thelephora, Piloderma, Russula, Lactarius, Cortinarius, Hebeloma, Tuber, Wilcoxina and others. Basidiomycetes Piloderma bicolor and P. sphaerosporum as well as ascomycetes from genera Helvella, Tuber and Wilcoxina are well known as EcM fungi, which confirms the possibility of carbon inflow to the pyroloids through the fungal symbionts

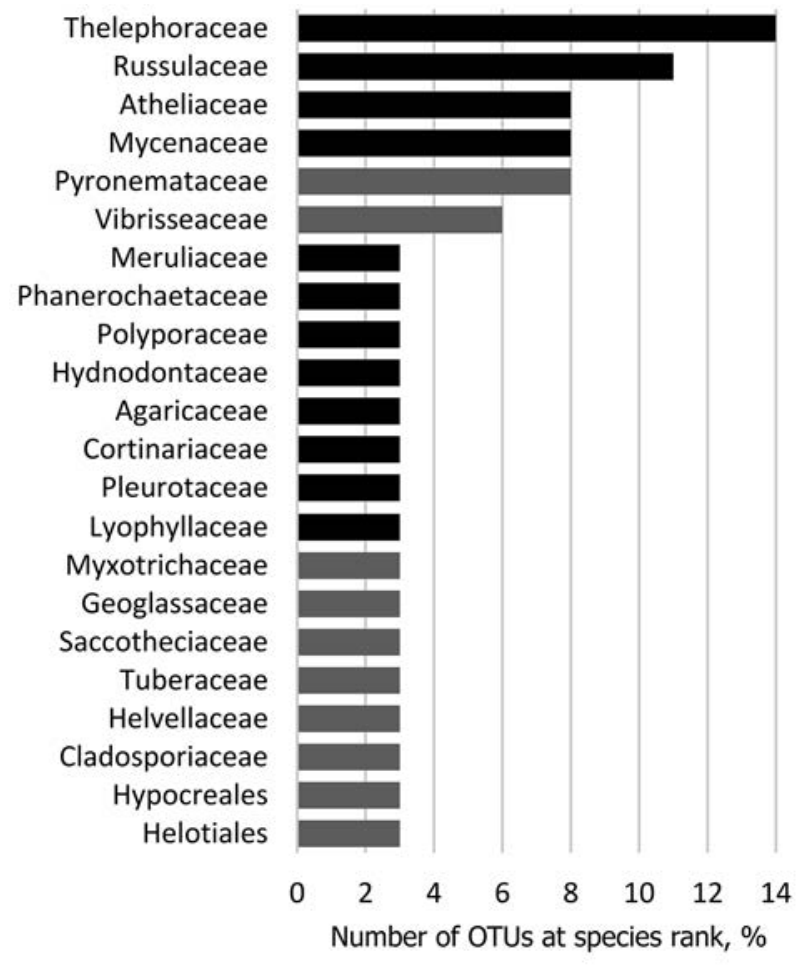

Figure 1 Relative abundance at family (and order) rank of ectomycorrhizal, putative ectomycorrhizal and saprotrophic fungi associated with three pyroloid species (Orthilia secunda, Pyrola rotundifolia and P. media). Black colour indicates basidiomycetes taxa, gray - ascomycetes taxa 


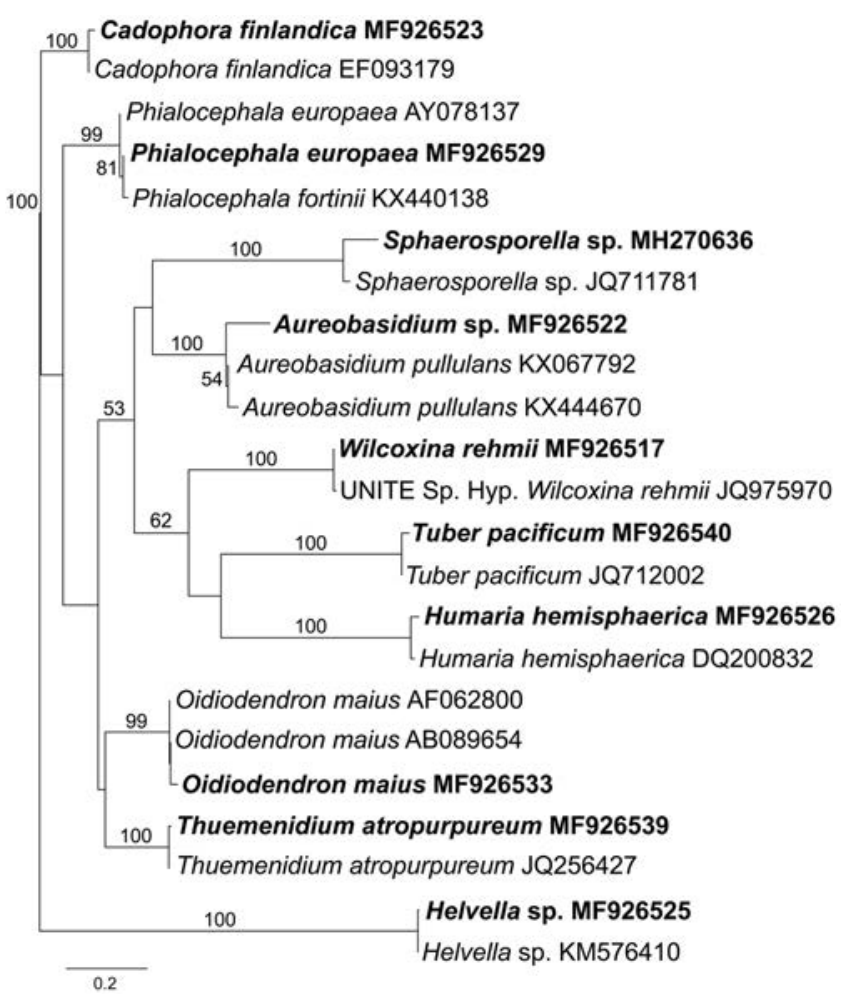

Figure 2 Phylogenetic placement of ascomycetes mycobionts from Pyrola rotundifolia, P. media and Orthilia secunda root systems detected in this study. Newly generated $n r$ ITS sequences (indicated by bold font) were complemented with reference sequences derived from GenBank and analyzed with maximum likelihood using PhyML. The tree is midpoint rooted. Branch support is given above the branches

common with photosynthetic wood plants (Rinaldi et al. 2008). The presence of mutual fungal partners was confirmed using molecular method in our study. The dominant mycobionts shared by both pyroloids and surrounding trees in three sites were: Piloderma sphaerosporum, Tomentella stuposa isolated from Orthilia secunda and Picea abies roots in S1, Russula velenovskyi - from $O$. secunda and Tilia cordata, Thelephora cf. anthocephala - from O. secunda and Populus tremula in S5, and Phialocephala fortinii - from O. secunda and Picea abies in $\mathrm{S} 1$ and S4. The fungal symbionts in these ternary functional complexes "mixotrophic plant-EcM fungus-woody plants" are for the most part not strictly specialized for a particular tree species. Therefore, it is apparent that the adult pyroloid plants are able to use a rather wide range of tree partners as their heterotrophic carbon source.

Most of the taxa revealed in our work as symbionts of the studied plants encompassed those already identified for other pyroloids (Pyrola and Orthilia) in Europe and North America, corroborating data from similar studies (Tedersoo et al. 2007, Zimmer et al. 2007, Massicotte et al. 2008, Vincenot et al. 2008, Hynson \& Bruns 2009, Toftegaard et al. 2010). This is the evidence for the pyroloid species tendency to associate with similar fungal taxa, regardless of plants geographical distribution. However, a large group of EcM fungi from the order Sebacinales forming mycorrhiza with pyroloids at the early (heterotrophic) stage of their development (Hynson et al. 2013), was not detected in our study.
Although mycorrhizal fungal symbionts play a crucial role in the pyroloids performance, precious few is known about their interactions with potentially diverse communities of so-called root-associated fungi (RAF) or endophytes. At present, evidence is beginning to accumulate that they may act as EcM fungi or partners in ericoid mycorrhizas (Rinaldi et al. 2008). We found three OTUs belonging to RAF community, among them were Phialocephala fortinii and $P$. europaea capable of forming stable but nonspecific connections with roots of many plant species (Queloz et al. 2005). The taxa detected from Pyrola media root system also included a provisional endophyte, Cadophora finlandica, whose ability to form ericoid mycorrhiza and ectomycorrhiza has been reported previously in several studies (Gorfer et al. 2007, Peterson et al. 2008).

For some fungal taxa identified, the association with the roots of pyroloids has been revealed for the first time: ascomycetes - Phialocephala europaea, Oidiodendron maius, Thuemenidium atropurpureum, Aureobasidium sp., Tuber pacificum, Sphaerosporella sp. and Helvella sp., and basidiomycetes Bjerkandera adusta, Cortinarius hemitrichus, Echinoderma asperum, Gerhardtia borealis, Lactarius torminosus, Lentinus crinitus, Luellia recondita, Mycena galopus, $M$. cf. cinerella, $M$. cf. citrinomarginata, Phanerochaete chrysosporium, Pleurotus ostreatus, Pseudotomentella tristis, Russula cf. subfoetens, Tylospora fibrillosa. For some of them, the ecological role is quite apparent (for example, for Cortinarius, Russula, Lactarius, Tuber, Helvella, Tylospora, which are typical EcM fungi), but a function of other taxa in the plant community remains a mystery. In the present study, we can only register, for the time being, the fact of their presence in the roots of the studied plants and hypothesize their involving into the arbutoid mycorrhiza forming. To make more reliable conclusions about their relationship with plants, more research is necessary.

The most common species Oidiodendron maius, a wellknown symbiont in ericoid mycorrhizas (Read 1996), identified in the present study, was only once detected from pyroloids roots (Pyrola morrisonensis, Matsuda et al. 2017). It has to be born in mind that, as yet, we have no clear explanation for the nature of the relationship between symbionts and hosts in ericoid mycorrhizas (Rice \& Currah 2006): whether this is true mutualistic interrelation, or the plant acts as a preventive refugia for fungus, or fungus parasitizes the plant, or there is a certain combination of those three states. In vitro experiments display that $O$. maius can improve the growth of host plants by facilitating their nutrition (preferably nitrogen) and detoxifying the soil environment (Rice \& Currah 2006, Daghino et al. 2016), though the feedback benefit for O. maius is not so obvious. This fungal species possesses prominent enzyme systems (Wei et al. 2016) and can decompose complex polymer compounds in soil, therefore it is highly unlikely that the fungus rely entirely on its photosynthetic host for surviving. Nevertheless, the fungus may obtain some products of photosynthesis in addition to saprotrophic carbon, that potentially gives $O$. maius a competitive advantage over other soil-inhabiting fungi. The question of the advantages for both partners under particular environmental conditions that determine the functional nature of these relations is still open. The tendency to microspermy 


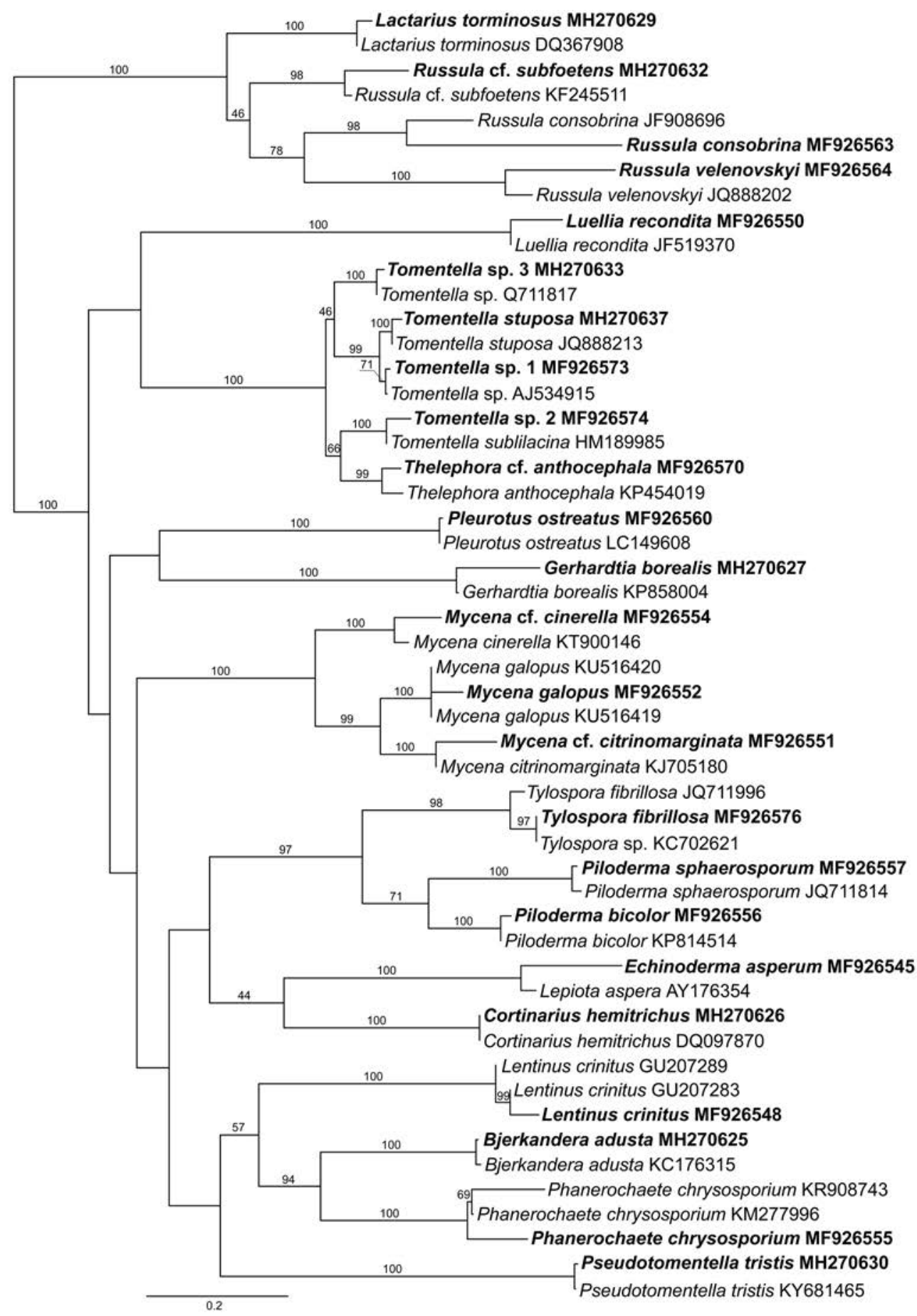

Figure 2 Phylogenetic placement of basidiomycetes mycobionts from Pyrola rotundifolia, P. media and Orthilia secunda root systems detected in this study. Newly generated $n$ rITS sequences (indicated by bold font) were complemented with reference sequences derived from GenBank and analyzed with maximum likelihood using PhyML. The tree is midpoint rooted. Branch support is given above the branches

in Ericaceae as well as saprotrophic ability of its fungal partners (defined as endophytes or symbionts) indicate that ericoid mycorrhizal associations may represent another example of fungal parasitism controlled by host plant similar to one observed in orchids. Such plants taking O. maius into their functional area gain a competitive advantage in plant community by increasing supply of organic matter inaccessible to other plants. Given the wide taxonomic tolerance that ericoids show towards endophytic fungi in vitro (Rice \& Currah 2006), there is an obvious need for detailed ex- amination of endophytic fungi diversity in situ and enigmatic ecological role of Myxotrichaceae, Helotiales and close taxa.

A very interesting result of our work is a repeated discovery (in six samples) of Thuemenidium atropurpureum from roots of Pyrola media and Orthilia secunda in three study sites (S1, S3, S4). This species from the family Geoglossaceae s.l. is usually regarded as typical inhabitant of pastures or meadows on sandy or acidic soils, and rarely occurs in woodland (Kučera et al. 2008). Up to now, no obvious ecological connection of this species with any groups of plants was 
observed, and its function in plant communities was not clarified. Alternatively, it is known that the closest species, Sabuloglossum arenarium, growing under similar ecological conditions, is associated with plants from Empetraceae and Ericaceae (Ericales) (Beenken \& Horn 2008). To our knowledge, one of the characteristics of fungi that form eryticoid mycorrhizas is their ability to enhance the availability of $\mathrm{N}$ for plants (Read 1996). Most plants can consume nitrogen in two forms - nitrate $\left(\mathrm{NO}_{3}^{-}\right)$or ammonium $\left(\mathrm{NH}_{4}^{+}\right)$. Some laboratory experiments (Rosen et al. 1990, Smith \& Read 2008) demonstrated that many members of Ericales are not capable of consuming $\mathrm{NO}_{3}^{-}$as a source of nitrogen. This is accounted for by the adaptation of given plants to acidic soil habitats (with $\mathrm{pH}$ in the range 4-5.5), where the nitrate form of $\mathrm{N}$ is poorly represented and therefore not available to plants. Recent works (Kosola et al. 2007, Yin et al. 2010) showed that an inoculation of ericoids with appropriate symbiotic fungi (e.g. Pezoloma ericae) significantly increases the ability of plants to adsorbtion of nitrogen in the form of nitrates and provides an advantage in growth over other competitors in certain environmental conditions. This provides the opportunity to propose that, in our case, there is a similar situation observed, when T. atropurpureum may play an important role in helping the pyroloids to use more effectively organic and mineral resources from strongly acidic soils under coniferous (Picea and Pinus) forests.

In the roots and rhizomes of the studied model species, we found quite a lot of saprotrophic basidiomycetes (from genera Bjerkandera, Gerbardtia, Luellia, Phanerochaete, Lentinus, Echinoderma and Pleurotus). All species from the genus Mycena were commonly interpreted previously as saprotrophs, though some were also registered occasionally as mycobionts in Pyrola media roots (Toftegaard et al. 2010) or as symbionts of orchid mycorrhizas (Ogura-Tsujita et al. 2009, Hashimoto et al. 2012). This fact may indicate that there is a general mixotrophic strategy for green orchids and pyroloids with similar suite of fungal symbionts, although the connection with certain taxa of fungi is more obligate for orchids. Despite free-living saprotrophs can have part in forming orchid mycorrhizas, it is most likely that the fungi we revealed in the present study may represent potential partners of ectomycorrhizas, and a closer examination of the surrounding trees roots is required.

Future research, no doubt, will clarify the ecological role of many fungi presently detected as well as their relations with pyroloid plants.

\section{ACKNOWLEDGEMENTS}

The authors are sincerely grateful to Zhiguli Nature Reserve and Zvenigorod Biological Station staff for their assistance in field research. The research was financially supported by the Russian Foundation for Basic Researches, project No. 16-04-01468 (to E. Malysheva, V. Malysheva, E. Voronina, A. Kovalenko - probes sampling, molecular research). Partial financial support by the Russian Science Foundation (RSCF), programme 14-50-00029, to E. Voronina (macrofungal species morphological identification and herbarization) is acknowledged.

\section{LITERAT URE CITED}

Beenken, L. \& K. Horn 2008. Erstnachweis von Geoglossum arenarium am Großen Arber im Bayerischen Wald. Bavarian Forest. Zeitschrift für Mykologie 74(1):119-126.

Bidartondo, M.I., B. Burghardt, G. Gebauer, T.D. Bruns \& D.J. Read 2004. Changing partners in the dark: isotopic and molecular evidence of ectomycorrhizal liaisons between forest orchids and trees. Proceedings of the Royal Society Biological Sciences B: Biological Sciences 271:1799-1806.

Daghino, S., E. Martino \& S. Perotto 2016. Model system to unravel the molecular mechanisms of heavy metal tolerance in the ericoid mycorrhizal symbiosis. Mycorrbiza 26: 263-274.

Edgar, R.C. 2004. MUSCLE: multiple sequence alignment with high accuracy and high throughput. Nucleic Acids Research 32(5):1792-1797.

Eriksson, O. \& K. Kainulainen 2011. The evolutionary ecology of dust seeds. Perspectives in Plant Ecology Evolution and Systematics 13: 73-87.

Erland, S. 1995. Abundance of Tylospora fibrillosa ectomycorrhizas in a South Swedish spruce forest measured by RFLP analysis of the PCR-amplified rDNA ITS region. Mycological Research 99(12):1425-1428.

Freudenstein, J.V. 1999. Relationships and character transformation in Pyroloideae (Ericaceae) based on ITS sequences, morphology, and development. Systematic Botany 24:398-408.

Gardes, M. \& T.D. Bruns 1993. ITS primers with enhanced specificity for basidiomycetes - application to the identification of mycorrhizae and rusts. Molecular Ecology 2:113-118.

Gorfer, M., S. Klaubauf, D. Bandian \& J. Strauss 2007. Cadophora finlandia and Phialocephala fortiniz: Agrobacterium-mediated transformation and functional GFP expression. Mycological Research 111: 850-855.

Hashimoto, Y., S. Fukukawa, A. Kunishi, H. Suga, F. Richard, M. Sauve \& M.-A. Selosse 2012. Mycoheterotrophic germination of Pyrola asarifolia dust seeds reveals convergences with germination in orchids. New Phytologist 195: 620-630.

Hynson, N.A. \& T.D. Bruns 2009. Evidence of a mycoheterotroph in the plant family Ericaceae that lacks mycorrhizal specificity. Proceedings of the Royal Society Biological Sciences B: Biological Sciences 276:4053-4059.

Hynson, N.A., K. Preiss, G. Gebauer \& T.D. Bruns 2009. Isotopic evidence of full and partial myco-heterotrophy in the plant tribe Pyroleae (Ericaceae). New Phytologist 182:719-726.

Hynson, N.A., M. Weiss, K. Preiss, G. Gebauer \& K.K. Treseder 2013. Fungal host specificity is not a bottleneck for the germination of Pyroleae species (Ericaceae) in a Bavarian forest. Molecular Ecology 22(5):1473-1481.

Johansson, V.A., A. Mikusinska, A. Ekblad \& O. Eriksson 2015. Partial mycoheterotrophy in Pyroleae: nitrogen and carbon stable isotope signatures during development from seedling to adult. Oecologia 177:203-211.

Kosola, K.R., B.A.A. Workmaster \& P.A. Spada 2007. Inoculation of cranberry (Vaccinium macrocarpon) with the ericoid mycorrhizal fungus Rhizoscyphus ericae increases nitrate influx. New Phytologist 176:184-196.

Kõljalg, U., K.-H. Larsson, K. Abarenkov, R.H. Nilsson, I.J. Alexander, U. Eberhardt et al. 2005. UNITE: a database providing web-based methods for the molecular identification of ectomycorrhizal fungi. New Phytologist 166:1063-1068. 
Kõljalg, U., R.H. Nilsson, K. Abarenkov, L. Tedersoo, A.F.S. Taylor \& 37 others 2013. Towards a unified paradigm for sequence-based identification of fungi. Molecular Ecology 22:5271-5277.

Kron, K.A., W.S. Judd, P.F. Stevens, D.M. Crayn, A.A. Anderberg, P.A. Gadek, C.J. Quinn \& J.L. Luteyn 2002. Phylogenetic classification of Ericaceae: molecular and morphological evidence. Botanical Review 68:335-423.

Kučera, V., P. Lizoň \& I. Kautmanová 2008. Geoglossaceous fungi in Slovakia: rare and new taxa for the territory. Biologia 63(4): 482-486.

Leake, J.R. 1994 Tansley review No. 69. The biology of myco-heterotrophic ('saprophytic') plants. New Phytologist 127:171-216.

Liu, Z.W., J. Zhou, E.D. Liu \& H. Peng 2010. A molecular phylogeny and a new classification of Pyrola (Pyroleae, Ericaceae). Taxon 59:1690-1700.

Malysheva, E.F., V.F. Malysheva, A.E. Kovalenko, E.A. Pimenova, M.N. Gromyko, S.N. Bondarchuk \& E.Yu. Voronina 2016. Below-ground ectomycorrhizal community structure in the postfire successional Pinus koraiensis forests in the Central Sikhote-Alin (the Russian Far East). Botanica Pacifica 5(1):19-31.

Massicotte, H.B., L.H. Melville, R. Molina \& R.L. Peterson 1993. Structure and histochemistry of mycorrhizae synthesized between Arbutus menziesii (Ericaceae) and two basidiomycetes, Pisolithus tinctorius (Pisolithaceae) and Piloderma bicolor (Corticiaceae). Mycorrbiza 3(1):1-11.

Massicotte, H.B., L.H. Melville, L.E. Tackaberry \& R.L. Peterson 2008. A comparative study of mycorrhizas in several genera of Pyroleae (Ericaceae) from western Canada. Botany 86:610-622.

Matsuda, Y., S. Shimizu, M. Mori, S.I. Ito \& M.A. Selosse 2012. Seasonal and environmental changes of mycorrhizal associations and heterotrophy levels in mixotrophic Pyrola japonica (Ericaceae) growing under different light environments. American Journal of Botany 99:1177-1188.

Matsuda, Y., T. Uesugi, T.-W. Hsu, C.-F. Chen 2017. Mycorrhizal fungi associated with Taiwanese Pyrola morrisonensis (Ericaceae) in a naturally regenerated forest. Taiwania 62(4):399-406.

Merckx, V. 2013. Mycoheterotrophy: the biology of plants living on fungi. Springer-Verlag, Berlin, $356 \mathrm{pp}$.

Ogura-Tsujita, Y., G. Gebauer, T. Hashimoto, H. Umata \& T. Yukawa 2009. Evidence for novel and specialized mycorrhizal parasitism: the orchid Gastrodia confusa gains carbon from saprotrophic Mycena. Proceedings of the Royal Society Biological Sciences B: Biological Sciences 276:761-767.

Queloz, V., C.R. Grünig, T.N. Sieber \& O. Holdenrieder 2005. Monitoring the spatial and temporal dynamics of a community of the tree-rot endophyte Phialocephala fortinii s.l. New Phytologist 168:651-660.

Peterson, R.L., C. Wagg \& M. Pautler 2008. Associations between microfungal endophytes and roots: do structural features indicate function? Botany 86:445-456.

Read, D.J. 1996. The structure and function of the ericoid mycorrhizal root. Annals of Botany 77:365-374.

Rice, A.V. \& R.S. Currah 2006. Oidiodendron maius: saprobe in sphagnum peat, mutualist in ericaceous roots? In: Microbial Roots Endophytes (B.J.E. Shulz, C.J.C. Boyle \& T.N. Sieber, eds.), pp. 227-246, Springer, NY.
Rinaldi, A.C., O. Comandini \& T.W. Kuyper 2008. Ectomycorrhizal fungal diversity: separating the wheat from the chaff. Fungal Diversity 33:1-45.

Robertson, D.C. \& J.A. Robertson 1985. Ultrastructural aspects of Pyrola mycorrbizae. Canadian Journal of Botany 63: 1089-1098.

Rosen, C.J., D.L. Allan \& J.J. Luby 1990. Nitrogen form and solution $\mathrm{pH}$ influence growth and nutrition of two Vaccinium clones. Journal of the American Society for Horticultural Sciience 115:83-89.

Selosse, M.-A. \& M. Roy 2009. Green plants that feed on fungi: facts and questions about mixotrophy. Trends in Plant Sciences 14:64-70.

Smith, S.E. \& D.J. Read 2008. Mycorrbizal Symbiosis, 3rd edition, Academic Press, NY, 787 pp.

Smith, M.E., A. Gryganskyi, G. Bonito, E. Nouhra, B. Moreno-Arroyo \& G. Benny 2013. Phylogenetic analysis of the genus Modicella reveals an independent evolutionary origin of sporocarp-forming fungi in the Mortierellales. Fungal Genetics and Biology 61:61-68.

Tamura, K., G. Stecher, D. Peterson, A. Filipski \& S. Kumar 2013. MEGA6: molecular evolutionary genetics analysis version 6.0. Molecular Biology and Evolution 30:2725-2729.

Tedersoo, L., P. Pellet, U. Kõljalg \& M.-A. Selosse 2007. Parallel evolutionary paths to mycohetereotrophy in understorey Ericaceae and Orchidaceae: ecological evidence for mixotrophy in Pyroleae. Oecologia 151:206-217.

Tedersoo, L., T.W. May \& M.E. Smith 2010. Ectomycorrhizal lifestyle in fungi: global diversity, distribution, and evolution of phylogenetic lineages. Mycorrbiza 20:217-263.

Toftegaard, T., G.R. Iason, I.J. Alexander, S. Rosendahl \& A.F.S. Taylor 2010. The threatened plant intermediate wintergreen (Pyrola media) associates with a wide range of biotrophic fungi in native Scottish pine woods. Biodiversity and Conservation 19:3963-3971.

Vincenot, L., L. Tedersoo, F. Richard, H. Horcine, U. Kõljalg \& M.A. Selosse 2008. Fungal associates of Pyrola rotundifolia, a mixotrophic Ericaceae, from two Estonian boreal forests. Mycorrbiza 19:15-25.

Wei, X., J. Chen \& D. Pan 2016. A new Oidiodendron maius strain isolated from Rhododendron fortunei and its effects on nitrogen uptake and plant growth. Frontiers in Microbiology $7: 1327$.

White, T.J., T.D. Bruns, S. Lee \& J. Taylor 1990. Amplification and direct sequencing of fungal ribosomal RNA genes for phylogenetics. In: PCR Protocols. A guide to methods and applications (M.A. Innis, D.H. Gelfand, J.J. Sninsky \& T.J. White, eds), pp. 315-322, New York.

Yin, L., C. Zhang \& B. Yang 2010. Characteristics of nitrogen absorbed by ericoid mycorrhizal fungi and impact on growth of Rhododendron fortunei. Scientia Agricultura Sinica 43:868-872.

Zimmer, K., N.A. Hynson, G. Gebauer, E.B. Allen, M.F. Allen \& D.J. Read 2007. Wide geographical and ecological distribution of nitrogen and carbon gains from fungi in pyroloids and monotropoids (Ericaceae) and in orchids. New Phytologist 175:166-175. 